\title{
THE RADIUS OF CONVEXITY FOR A SPECIAL CLASS OF MEROMORPHIC FUNCTIONS
}

BY DOROTHY BROWNE SHAFFER

Communicated by E. Isaacson, June 15, 1972

Let $\Sigma$ denote the class of functions $F(\zeta)=\zeta+a_{0}+a_{1} / \zeta+\cdots$ regular in $1<|\zeta|<\infty$. In this paper the radius of convexity for the subclass $\Sigma_{\alpha}$ defined by the additional condition $\operatorname{Re} F^{\prime}(\zeta)>\alpha$, where $0 \leqq \alpha<1$, is determined. The results are sharpened for functions with missing terms in the expansion. The proofs are based on inequalities for analytic functions established by the author [3]. The functions $F(\zeta)$ are not assumed to be schlicht; in fact, the extremal functions for $\alpha<\frac{1}{2}$ will not be schlicht. It is not known whether the univalence of $F(\zeta)$ follows from the condition $\operatorname{Re} F^{\prime}(\zeta)>\frac{1}{2}$ for $R_{c}>|\zeta|>R>1$. The radius of convexity $\left(R_{c} \sim 1.78\right)$ for the class $\Sigma$ with the assumption of schlichtness is due to Goluzin $\left[1\right.$, p. 136]; Robertson [2, Theorem 4] found $R_{c}=3^{1 / 2}$ for the subclass of schlicht and starlike functions. It will be shown that: for the class $\Sigma_{1 / 2}, R_{c}=3^{1 / 2}$; and $R_{c}<3^{1 / 2}$ for $\alpha>\frac{1}{2}$. by

THEOREM 1. The radius of convexity, $R_{0}$, for functions $F(\zeta) \in \Sigma_{\alpha}$ is given

$$
R_{0}^{2} \leqq\left\{\left[(3+c)^{2}+4 c\right]^{1 / 2}+(3+c)\right\} / 2
$$

where $c=1-2 \alpha$.

Proof. Let

$$
h(z) \equiv F^{\prime}(1 / z)=1+b_{1} z^{2}+\cdots .
$$

From [4, Theorem 7], we have

$$
\left|\frac{h^{\prime}(z)}{h(z)}\right| \leqq \frac{2(1+c)|z|}{\left(1+c|z|^{2}\right)\left(1-|z|^{2}\right)} \text { for }|z|<1 .
$$

By differentiation of (2) we obtain

$$
z h^{\prime}(z) / h(z)=-\zeta F^{\prime \prime}(\zeta) / F^{\prime}(\zeta)
$$

The condition for convexity $\operatorname{Re}\left(\zeta F^{\prime \prime}(\zeta) / F^{\prime}(\zeta)+1\right) \geqq 0$ will be satisfied if

$$
2(1+c)|z|^{2} \leqq\left(1+c|z|^{2}\right)\left(1-|z|^{2}\right) .
$$

This is equivalent to $|\zeta|>R_{0}$.

Let $p^{0}(z)=\left(1+c z^{2}\right) /\left(1-z^{2}\right)$, then $F^{0}(\zeta)=\zeta+[(c+1) / 2][\log (\zeta-1) /(\zeta+1)]$

AMS (MOS) subject classifications (1969). Primary 3010.

Copyright (C) American Mathematical Society 1973 
will have $\operatorname{Re}\left(\zeta F^{\prime \prime}(\zeta) / F^{\prime}(\zeta)+1\right)=0$ for $\zeta=R_{0}$. We have $F^{0}(\zeta)=$ $\zeta-(c+1) / \zeta+\cdots$.

If $c>0$, i.e., $\alpha<\frac{1}{2},\left|a_{1}\right|>1$ and $F^{0}(\zeta)$ is not schlicht.

Corollary. For the special case, $\operatorname{Re} F^{\prime}(\zeta)>0$, we have $c=1$ and $R_{0}=\left[5^{1 / 2}+2\right]^{1 / 2} ;$ for $\operatorname{Re} F^{\prime}(\zeta)>\frac{1}{2}, c=0$ and $R_{0}=3^{1 / 2}$.

THEOREM 2. Let $F(\zeta) \in \Sigma_{\alpha}$ have the expansion $F(\zeta)=\zeta+a_{0}+a_{n} / \zeta^{n}+$ $a_{n+1} / \zeta^{n+1}+\cdots$ then the radius of convexity

$$
R_{0}^{n+1}=\left\{\left[(n+2+n c)^{2}+4 c\right]^{1 / 2}+[n+2+n c]\right\} / 2 .
$$

The proof is similar to Theorem 1, based on the inequality [4]

$$
\left|h^{\prime}(z) / h(z)\right| \leqq(1+c) n|z|^{n-1} /\left[1-(1-c)|z|^{n}-c|z|^{2 n}\right]
$$

for functions with expansion $h(z)=1+c_{n} z^{n}+\ldots, n \geqq 1$.

\section{BIBLIOGRAPHY}

1. G. M. Goluzin, Geometrische Funktionentheorie, Hochschulbücher für Mathematik, Band 31, VEB Deutscher Verlag der Wissenschaften, Berlin, 1957. MR 19, 735.

2. M. M. Robertson, Extremal problems for analytic functions with positive real part and applications, Trans. Amer. Math. Soc. 106 (1963), 236-253. MR 26 \#325.

3. Dorothy B. Shaffer, On bounds for the derivative of analytic functions, Proc. Amer. Math. Soc. (to appear).

4. - Distortion theorems for a special class of analytic functions, Proc. Amer. Math. Soc. (to appear).

Department of Mathematics, Fairfield University, Fairfield, Connecticut 06430 